\title{
Survey on Recognize Malignant Facebook Application
}

\author{
Kiran Bhise $^{1}$, R. S. Shishupal ${ }^{2}$ \\ ${ }^{1}$ M.E (Computer Network) Department of Computer Engineering, Sinhagad Institute of Technology, Lonavala, Pune, India. Savitribai \\ Phule Pune University, Pune, Maharashtra, India -411007 \\ ${ }^{2}$ Asst. Prof (Computer) Department of Computer Engineering, Sinhagad Institute of Technology, Lonavala, Pune, India. Savitribai Phule \\ Pune University, Pune, Maharashtra, India -411007
}

\begin{abstract}
With daily installs and use of third party apps are important reasons for the popularity and addictiveness of facebook. Hackers realized the potential of using apps for spreading spam and malware. Here the problem is already find out so it gives $13 \%$ of apps are malicious. So researchers are focused on detecting malicious posts and campaigns. Here question may arise that given a Facebook application, can we determine if it is malicious? So key is to developing REAppE- Facebook's Rigorous Application Evaluator is the first tool focused on detecting malicious apps on Facebook. In this paper we discussed the survey on different techniques used for malicious apps protection for facebook.
\end{abstract}

Keywords: Facebook Apps, Malicious Apps, Profiling Apps, Online Social Networks

\section{Introduction}

Online social networks (OSN) enable third party apps to enhance the user experience on the platforms. Such enhancement includes interesting or entertaining ways of communicating among online friends and different activities such as playing games or listening songs. If we take example, facebook provides developers an API that facilities app integration into Facebook user-experience. Recently, hackers have started taking advantage of the recognition of this third-party apps platform and deploying malicious applications. Malicious apps will give a profitable business for hackers, given the recognition of OSNs, with Facebook leading the method with $900 \mathrm{M}$ active users. There are some ways that hackers will get pleasure from a malicious app: (a) the app will reach large numbers of users and their friends to unfold spam, (b) the app can acquire users' personal data like email address, home town, and gender, and (c) the app will "re-produce" by creating different malicious apps standard. To form matters worse, the readying of malicious apps is simplified by ready-to-use toolkits beginning at $\$ 25$. In different words, there's motive and chance, and as a result, there are several malicious apps spreading on Facebook each day.

Despite the on top of worrisome trends, today, a user has terribly restricted information at the time of putting in associate app on Facebook. In other words, the matter is: given associate app's identity variety (the unique symbol assigned to the app by Facebook), will we have a tendency to observe if the app is malicious? Presently, there's no business service, publicly-available data, or research-based tool to advise a user concerning the risks of associate app. As we have a tendency to show in Sec. 3, malicious apps are widespread and that they simply unfold, as associate infected user jeopardizes the safety of all its friends. So far, the analysis community has paid very little attention to OSN apps specifically. Most analysis associated with spam and malware on Facebook has centered on detection malicious posts and social spam campaigns [6, 7, and 16]. A recent work studies however app permissions and community ratings correlate to privacy risks of Facebook apps [17]. Finally, there are some community-based feedback driven efforts to rank applications, like Whatapp [22]; although these may be terribly powerful within the future, thus far they need received little adoption.

In this paper, we have a tendency to gift a web spam filtering system specifically designed for OSNs and may be deployed as a part of the OSN platform. Once the initial coaching phase, it with efficiency inspects the stream of user generated messages, at once dropping those classified as spam before they reach the meant recipients. The system owns four fascinating properties as a web filtering tool which are: i) high accuracy, ii) no would like for all campaigns to be gift within the coaching set, iii) no would like for frequent re-training, and iv) low latency. The key insight is that we have a tendency to forever look for to uncover the connection among all the messages by activity agglomeration on them, rather than directly inspecting every individual message while not correlating it with others. The related to spam messages type spam campaigns. Though the clustering approach has been used for offline spam analysis $[4,16]$, it's ne'er used for on-line spam filtering owing $t$ its process overhead. We have a tendency to leverage progressive agglomeration and parallelization to handle this challenge. When a new message is generated, the system organizes it, along with all the antecedently ascertained messages, into clusters. The new message is then classified in step with whether or not or not the cluster it resides in could be a spam cluster, which is determined by all the messages within the same cluster conjointly.

The system has 2 blessings over the attacker; user feedback and international data. User feedback is each specific and implicit. specific feedback includes mark as spam or news a user. Implicit feedback includes deleting a post or rejecting a devotee request. each implicit and specific feedback area unit valuable and central to defense. Additionally to user feedback, the system has data of combination patterns and

\section{Volume 4 Issue 12, December 2015}




\section{International Journal of Science and Research (IJSR) \\ ISSN (Online): 2319-7064 \\ Index Copernicus Value (2013): 6.14 | Impact Factor (2014): 5.611}

what's traditional and weird. This facilitates anomaly detection, clustering, and has aggregation. The system uses these 2 blessings in each detection and response. Some of the additional ancient machine learning metrics don't really apply to adversarial learning in our context, or a minimum of area unit less vital for instance, classifier accuracy. The graph is being defended across multiple synchronous attacks victimization finite resources. The goal is to guard the graph against all attacks rather than to maximize the accuracy of anyone specific classifier. The opportunity cost of purification a model for one attack could also be increasing the detection and response on different attacks. For these reasons, response and detection latencies will be additional vital than preciseness and recall. Even considering Associate in nursing attack in isolation, spending more time up a classifier will be problematic for 2 reasons. Injury accumulates quickly. Additional accounts get compromised and additional users get exposed to spam. A a pair of falsepositive rate nowadays on Associate in Nursing attack touching one, 000 users is healthier than a tenth falsepositive rate tomorrow on a similar attack touching one hundred,000 users. As well, as time progresses attacks change and coaching knowledge becomes less relevant. Done is commonly higher than excellent.

\section{Related Work}

Third-party applications (apps) drive the attractiveness of web and mobile application platforms. Several of those platforms adopt a decentralised management strategy, wishing on specific user consent for granting permissions that the apps request. Users got to swear totally on community ratings as the signals to spot the doubtless harmful and inappropriate apps even supposing community ratings generally reflect opinions regarding perceived practicality or performance rather than regarding risks. With the arrival of HTML5 web apps, such user-consent permission systems can become more widespread. we tend to study the effectiveness of user-consent permission systems through an outsized scale information assortment of Facebook apps, Chrome extensions and humanoid apps. Our analysis confirms that the present sorts of community ratings employed in app markets these days aren't reliable indicators of privacy risks of Associate in Nursing app. They discover some proof indicating makes an attempt to mislead or provoke users into granting permissions: free applications and applications with mature content request a lot of permissions than is typical; "lookalike" applications that have names kind of like fashionable applications also request a lot of permissions than is typical. We also realize that across all 3 platforms fashionable applications request a lot of permissions than average[4].

Online social networks (OSNs) area unit extraordinarily fashionable among net users. sadly, within the wrong hands, they are additionally effective tools for capital punishment spam campaigns. In this paper, author tend to gift a web spam filtering system that can be deployed as a element of the OSN platform to examine messages generated by users in time period. They propose to reconstruct spam messages into campaigns for classification rather than examine them separately. Though campaign identification has been used for offline spam analysis, we apply this method to help \{the online then we tend tob the net\} spam detection problem with sufficiently low overhead. Consequently, our system adopts a collection of novel options that effectively distinguish spam campaigns. It drops messages classified as "spam" before they reach the meant recipients, therefore protective them from varied sorts of fraud. They tend to evaluate the system victimization 187 million wall posts collected from Facebook and seventeen million tweets collected from Twitter. In several parameter settings, truth positive rate reaches eighty. $9 \%$ while the false positive rate reaches zero. $19 \%$ within the best case. In addition, it stays correct for quite nine months when the initial coaching part. Once deployed, it will perpetually secure the OSNs while not the necessity for frequent re-training. Finally, tested on a server machine with eight cores (Xeon E5520 2.2Ghz) and 16GB memory, the system achieves associate average outturn of 1580 messages/sec and a median processing latency of twenty one. $5 \mathrm{~ms}$ on the Facebook dataset.

Online social networks (OSNs) are well-liked collaboration and communication tools for several users and their friends. Sadly, in the wrong hands, they're conjointly effective tools for execution spam campaigns and spreading malware. Intuitively, a user is additional seemingly to retort to a message from a Facebook friend than from a alien, so creating social spam a simpler distribution mechanism than ancient email. In fact, existing proof shows malicious entities are already trying to compromise OSN account credentials to support these "high-return" spam campaigns. In this paper, author have a tendency to gift associate degree initial study to quantify and characterize spam campaigns launched victimization accounts on on-line social networks. They have a tendency to study an outsized anonymized dataset of asynchronous "wall" messages between Facebook users. They have a tendency to analyze all wall messages received by roughly three.5 million Facebook users (more than 187 million messages in all), and use a group of machine-driven techniques to sight and characterize coordinated spam campaigns. Their system detected roughly two hundred malicious wall posts with embedded URLs, originating from quite fifty seven, user accounts. They find that quite seventieth of all malicious wall posts advertise phishing sites. They have a tendency to conjointly study the characteristics of malicious accounts, and see that quite ninety seven ar compromised accounts, instead of "fake" accounts created alone for the aim of spamming. Finally, they observe that, once adjusted to the time of the sender, spamming dominates actual wall post activity within the early morning hours, once traditional users are asleep[7].

Online social networks (OSNs) became the new vector for crime, and hacker's area unit finding new ways that to propagate spam and malware on these platforms, which we check with as socware. As author have a tendency to show here, socware cannot be known with existing security mechanisms (e.g., URL blacklists), as a result of it exploits completely different weaknesses and infrequently has completely different intentions. In this paper, author have a tendency to gift MyPageKeeper, a Facebook application that they have developed to shield Facebook users from socware. Here, they have a tendency to gift results from the angle of over $12 \mathrm{~K}$ users WHO have put in MyPageKeeper and their roughly two.4 million friends. Their work makes 3 main

\section{Volume 4 Issue 12, December 2015}




\section{International Journal of Science and Research (IJSR) \\ ISSN (Online): 2319-7064 \\ Index Copernicus Value (2013): 6.14 | Impact Factor (2014): 5.611}

contributions. First, to alter protection of users at scale, they have a tendency to style AN economical socware detection technique that takes advantage of the social context of posts. They discover that our classifier is each correct $(97 \%$ of posts flagged by it are so socware and it incorrectly flags solely zero. $005 \%$ of benign posts) and economical [16].

Second, they have a tendency to show that socware considerably differs from ancient email spam or web-based malware. For example, web site blacklists determine solely third of the posts flagged by MyPageKeeper, whereas twenty sixth of flagged posts purpose to malicious apps and pages hosted on Facebook (which no current antivirus or blacklist is meant to detect). Third, they quantify the prevalence of socware by analyzing roughly m40 million posts over four months; forty ninth of our users were exposed to a minimum of one socware post during this amount. Finally, they determine a replacement variety of parasitic behavior, that they have a tendency to refer to as "Like-as-a-Service", whose goal is to by artificial means boost the number of "Likes" of a Facebook page.

Popular websites area unit under fire all the time from phishes, fraudsters, and spammers. They aim to steal user data and expose users to unwanted spam. The attackers have Brobdingnagian resources at their disposal. They're wellfunded, with full-time practiced labor, control over compromised and infected accounts, and access to global bonnets. Protective our users may be a difficult adversarial learning drawback with extreme scale and cargo needs. Over the past many years we've engineered and deployed a coherent, scalable, and protrusive real-time system to shield our users and the social graph. This system performs realtime checks and classifications on each browse and writes action. As of March 2011, this is often 25B checks per day, reaching $650 \mathrm{~K}$ per second at peak. The system additionally generates signals to be used as feedback in classifiers and different elements. we have a tendency to believe this technique has contributed to making Facebook the safest place on the web for individuals and their data. This paper outlines the look of the Facebook Immune System, the challenges we've featured and overcome, and the challenges we have a tendency to still face[17].

Due to the significance and vitalness of police work and suspending Twitter spammers, several researchers at the side of the engineers in Twitter Corporation have devoted themselves to keeping Twitter as spam-free on-line communities. Meanwhile, Twitter spammers also are evolving to evade existing detection techniques. During this paper, we make an empirical Associate in Nursingalysis of the evasion techniques used by Twitter spammers, and so style many new and strong options to notice Twitter spammers. Finally, we have a tendency to formalize the strength of twenty four detection options that are normally used within the literature moreover as our planned ones. Through their experiments, they have a tendency to show that our new designed options are effective to notice Twitter spammers, achieving a way higher detection rate than 3 progressive approaches whereas keeping Associate in Nursing even lower false positive rate [21]

With a lot of users tweeting round the world, real time search systems and differing kinds of mining tools square measure emerging to permit folks trailing the repercussion of events and news on Twitter. However, though appealing as mechanisms to ease the unfold of reports and permit users to debate events and post their standing, these services open opportunities for new types of spam. Trending topics, the most talked regarding things on Twitter at a given purpose in time, have been seen as a chance to come up with traffic and revenue. Spammers post tweets containing typical words of a trending topic and URLs, typically obfuscated by computer address softeners that lead users to utterly unrelated websites. This kind of spam will contribute to de-value real time search services unless mechanisms to fight and stop spammers may be found. In this paper we tend to take into account the matter of police investigation spammers on Twitter. we tend to initial collected an oversized dataset of Twitter that includes quite fifty four million users, 1.9 billion links, and nearly one. 8 billion tweets. Victimization tweets associated with 3 famous trending topics from 2009, we tend to construct an oversized labeled collection of users, manually classified into spammers and non-spammers. We tend to then establish variety of characteristics related to tweet content and user social behavior, which might doubtless be wont to discover spammers. We used these characteristics as attributes of machine learning process for classifying users as either spammers or no spammers. Our strategy succeeds at police investigation abundant of the spammers whereas solely little share of non-spammers are misclassified. more or less seventieth of spammers and ninety six of non-spammers were properly classified. Our results additionally highlight the foremost vital attributes for spam detection on Twitter[22].

Table 1: Survey Table

\section{Volume 4 Issue 12, December 2015}


International Journal of Science and Research (IJSR)

ISSN (Online): 2319-7064

Index Copernicus Value (2013): 6.14 | Impact Factor (2014): 5.611

\begin{tabular}{|c|c|c|c|c|}
\hline Author & Paper & Technique & Advantage & Disadvantages \\
\hline Yang et al. & $\begin{array}{c}\text { Die Free or Live Hard? } \\
\text { Empirical Evaluation and New } \\
\text { Design for } \\
\text { Fighting Evolving Twitter } \\
\text { Spammers }\end{array}$ & $\begin{array}{l}\text { To identify accounts of } \\
\text { spammers on Twitter }\end{array}$ & $\begin{array}{c}\text { It enables detection of } \\
\text { malicious apps that } \\
\text { propagate spam and malware } \\
\text { by luring normal } \\
\text { Users to install them. }\end{array}$ & $\begin{array}{l}\text { Process is to difficult to } \\
\text { implement. }\end{array}$ \\
\hline Chia et al. & $\begin{array}{c}\text { Is this App Safe? A Large Scale } \\
\text { Study on Application } \\
\text { Permissions and Risk Signals }\end{array}$ & $\begin{array}{c}\text { Investigated the } \\
\text { privacy intrusiveness of } \\
\text { Facebook apps and concluded } \\
\text { that currently available signals } \\
\text { such as community ratings, } \\
\text { popularity, and } \\
\text { external ratings }\end{array}$ & $\begin{array}{c}\text { It quantify the prevalence of } \\
\text { malicious apps, and develop } \\
\text { tools to identify malicious } \\
\text { apps. }\end{array}$ & $\begin{array}{l}\text { As user increases complexity } \\
\text { increases. }\end{array}$ \\
\hline Stein et al. & Facebook Immune System & $\begin{array}{l}\text { a scalable real-time adversarial } \\
\text { learning system } \\
\text { deployed in Facebook to protect } \\
\text { users from malicious activities }\end{array}$ & $\begin{array}{c}\text { It appears that Facebook has } \\
\text { recently } \\
\text { softened their controls for } \\
\text { handling spam apps }\end{array}$ & $\begin{array}{l}\text { It has not attracted many } \\
\text { reviews to date. }\end{array}$ \\
\hline
\end{tabular}

\section{Architectural View}

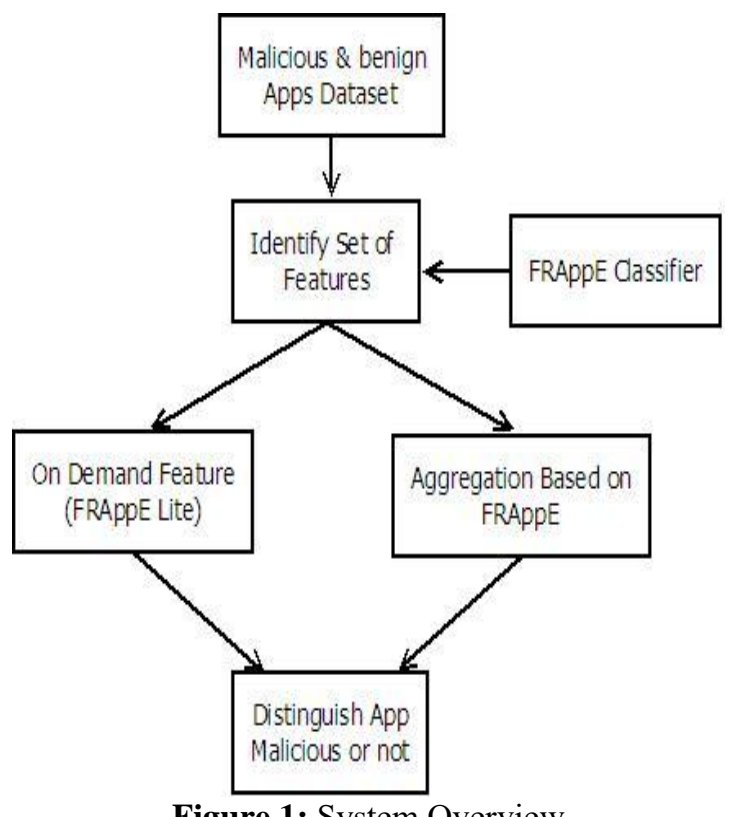

Figure 1: System Overview

\section{Conclusion}

Applications gift a convenient means that for hackers to unfold malicious content on Facebook. However, very little is known regarding the characteristics of malicious apps and the way they operate. In this work, employing a giant corpus of malicious Facebook apps discovered over a 9 month amount, we have a tendency to show that malicious apps dissent significantly from benign apps with relevancy many options. For example, malicious apps are rather more doubtless to share names with other apps, and that they generally request fewer permissions than benign apps. Investment our observations, we have a tendency to developed FRAppE, an accurate classifier for detective work malicious Facebook applications. Most apparently, we have a tendency to highlight the emergence of AppNetslarge teams of tightly connected applications that promote every other. we are going to still dig deeper into this system of malicious apps on Facebook, and that we hope that Facebook can profit from our recommendations for reducing the menace of hackers on their platform.

\section{References}

[1] F. Benevenuto, G. Magno, T. Rodrigues, and V. Almeida. Detecting spammers on Twitter. In CEAS, 2010.

[2] A. Besmer, H. R. Lipford, M. Shehab, and G. Cheek. Social applications: exploring a more secure framework. In SOUPS, 2009.

[3] C.-C. Chang and C.-J. Lin. LIBSVM: A library for support vector machines. ACM Transactions on Intelligent Systems and Technology, 2, 2011.

[4] P. Chia, Y. Yamamoto, and N. Asokan. Is this app safe? A large scale study on application permissions and risk signals. In WWW, 2012.

[5] F. J. Damerau. A technique for computer detection and correction of spelling errors. Commun. ACM, 7(3), Mar. 1964.

[6] H. Gao, Y. Chen, K. Lee, D. Palsetia, and A. Choudhary. Towards online spam filtering in social networks. In NDSS, 2012.

[7] H. Gao, J. Hu, C. Wilson, Z. Li, Y. Chen, and B. Y. Zhao. Detecting and characterizing social spam campaigns. In IMC, 2010.

[8] M. Gjoka, M. Sirivianos, A. Markopoulou, and X. Yang. Poking facebook: characterization of osn applications. In Proceedings of the first workshop on Online social networks, WOSN, 2008.

[9] J. King, A. Lampinen, and A. Smolen. Privacy: Is there an app for that? In SOUPS, 2011.

[10] A. Le, A. Markopoulou, and M. Faloutsos. Phishdef: Url names say it all. In Infocom, 2010.

[11] K. Lee, J. Caverlee, and S. Webb. Uncovering social spammers: social honeypots + machine learning. In SIGIR, 2010.

[12]S. Lee and J. Kim. Warningbird: Detecting suspicious urls in twitter stream. In NDSS, 2012.

[13] Y. Liu, K. P. Gummadi, B. Krishnamurthy, and A. Mislove. Analyzing facebook privacy settings: user expectations vs. reality. In IMC, 2011.

[14] J. Ma, L. K. Saul, S. Savage, and G. M. Voelker. Beyond blacklists: learning to detect malicious web sites from suspicious urls. In KDD, 2009.

[15] A. Makridakis, E. Athanasopoulos, S. Antonatos, D. Antoniades, S. Ioannidis, and E. P. Markatos. Understanding the behavior of malicious applications in 


\section{International Journal of Science and Research (IJSR) \\ ISSN (Online): 2319-7064}

Index Copernicus Value (2013): 6.14 | Impact Factor (2014): 5.611

social networks. Netwrk. Mag. of Global Internetwkg., 2010.

[16]M. S. Rahman, T.-K. Huang, H. V. Madhyastha, and M. Faloutsos. Efficient and Scalable Socware Detection in Online Social Networks. In USENIX Security, 2012.

[17] T. Stein, E. Chen, and K. Mangla. Facebook immune system. In Proceedings of the 4th Workshop on Social Network Systems, 2011.

[18] G. Stringhini, C. Kruegel, and G. Vigna. Detecting spammers on social networks. In ACSAC, 2010.

[19]K. Thomas, C. Grier, J. Ma, V. Paxson, and D. Song. Design and Evaluation of a Real-Time URL Spam Filtering Service. In Proceedings of the IEEE Symposium on Security and Privacy, 2011.

[20]N. Wang, H. Xu, and J. Grossklags. Third-party apps on facebook: privacy and the illusion of control. In CHIMIT, 2011.

[21]C. Yang, R. Harkreader, and G. Gu. Die free or live hard? empirical evaluation and new design for fighting evolving twitter spammers. In RAID, 2011.

[22] S. Yardi, D. Romero, G. Schoenebeck, et al. Detecting spam in a twitter network. First Monday, 2009. 